\title{
La evolución de los cuerpos académicos y de los investigadores registrados en el SNI de la Universidad Autónoma del Estado de México, periodo: 2002 - 2017
}

\section{The evolution of academic bodies and researchers registered in the SNI of the Autonomous University of the State of Mexico, period: 2002 - 2017}

\author{
CARRETO-BERNAL, Fernando $\dagger^{* *}$ \& RAMÍREZ-CARBAJAL, Alfredo Ángel“" \\ 'Facultad de Geografía. UAEMéx Instituto Literario 100, Centro, 50000 Toluca de Lerdo, Méx. \\ "Instituto de Estudios Sobre la Universidad UAEMéx. Instituto Literario 100, Centro, 50000 Toluca de Lerdo, Méx. \\ ID $1^{\text {er }}$ Autor: Fernando, Carreto-Bernal \\ ID $1^{\text {er }}$ Coautor: Alfredo Ángel, Ramírez-Carbajal
}

DOI: $10.35429 / J U P .2019 .8 .3 .19 .22$

Recibido: 10 de Abril, 2019; Aceptado 28 de Junio, 2019

\begin{abstract}
Resumen
El Sistema Nacional de Investigadores (SNI) fue creado el 26 de julio de 1984 por acuerdo presidencial y publicado en el diario oficial de la federación. Se estableció con la finalidad de reconocer la labor de las personas dedicadas a producir conocimiento científico y tecnología, esta distinción simboliza la calidad y prestigio de las contribuciones científicas. Su propósito general es promover el desarrollo de las actividades relacionadas con la investigación para fortalecer su calidad, desempeño y eficiencia. Por lo tanto, este documento refleja la situación de los cuerpos académicos de la Universidad Autónoma del Estado de México (UAEMéx) y de los investigadores reconocidos por el SNI.
\end{abstract}

Cuerpo académico, Investigadores, SNI

\begin{abstract}
The National System of Researchers (NSR) was created on July 26,1984 by presidential decree and published in the official journal of the federation. It was established in order to recognize the work of those engaged in producing scientific knowledge and technology, this award symbolizes the quality and prestige of scientific contributions. Its overall purpose is to promote the development of research activities to enhance the quality, performance and efficiency. Therefore, this document reflects the status of the academic bodies of the Autonomous University of the State of Mexico (UAEMEX) and researchers recognized by NSR.
\end{abstract}

Academic body, Researchers, SNI

Citación: CARRETO-BERNAL, Fernando \& RAMÍREZ-CARBAJAL, Alfredo Ángel. La evolución de los cuerpos académicos y de los investigadores registrados en el SNI de la Universidad Autónoma del Estado de México, periodo: 2002 - 2017. Revista de Políticas Universitarias. 2019. 3-8: 19-22.

\footnotetext{
* Correspondencia del Autor (correo electrónico: posgradoarc@ hotmail.com)

$\dagger$ Investigador contribuyendo como primer autor.
} 


\section{Introducción}

Los cuerpos académicos de la (UAEMéx) están conformados por el conjunto de profesores investigadores que comparten una o más líneas de estudio, cuyo propósito y metas están destinados a la generación y/o aplicación de nuevos conocimientos.

Por otra parte, en relación a los cuerpos académicos, se encuentra el objetivo de los investigadores que son reconocidos por el Sistema Nacional de Investigación (SNI) y cuyo fin es el de promover y fortalecer, a través de la evaluación, la calidad de la investigación científica y tecnológica, así como la innovación que se produce en el país. Tiene como elemento fundamental incrementar la cultura, productividad, competitividad y el bienestar social. Para tal efecto, las áreas de conocimiento son: Agropecuarias, Salud, Naturales y Exactas, Sociales y Administrativas, Ingeniería y Tecnología, Educación, Humanidades y Artes.

El objetivo del presente documento es determinar si existe una asociación entre el número total profesores integrados al sistema nacional de investigadores y los cuerpos académicos, cuantificando un periodo -a partir de 2002 hasta 2017-, en la Universidad Autónoma del Estado de México. Cabe mencionar la importancia de este trabajo, observa que en dicha relación las condiciones relevantes que impactan en la calidad de la educación que se imparte debieran corresponder a los profesores de nuestra institución que pertenecen al SNI.

\section{Método}

El método que se usó en este análisis es el descriptivo, que se utiliza para recoger, organizar, resumir, presentar, analizar, generalizar los resultados de las observaciones. Este método implica la recolección y presentación sistemática de datos para dar una idea clara de una determinada situación. Esto es, describe cómo es y se manifiesta una situación o evento. En este caso revisamos el estatus de los cuerpos académicos de la UAEM. El diseño utilizado fue transversal, ya que es un tipo de investigación en un tiempo delimitado. De ahí que el periodo de estudio es 2002-2013. Asimismo, se hizo uso de la investigación documental argumentativa exploratoria.
La población y muestra con la que se trabajo fue la totalidad de las categorías de los cuerpos académicos, así como de los investigadores SNI de la citada universidad. El Instrumento de recopilación de datos, fue de elaboración propia es, decir, se diseñó ex profeso para agrupar lo las estadísticas que se aplicarían en el estudio. Por último, cabe mencionar que la técnica estadística, operada fue la correlación por el hecho de que en probabilidad y estadística, la correlación indica la fuerza y la dirección de una relación lineal y proporcionalidad entre dos variables estadísticas.

Se considera que dos variables cuantitativas están correlacionadas cuando los valores de una de ellas varían sistemáticamente con respecto a los valores homónimos de la otra: si tenemos dos variables (A y B) existe correlación si al aumentar los valores de A lo hacen también los de $\mathrm{B}$ y viceversa. La correlación entre dos variables no implica, por sí misma, ninguna relación de causalidad.

\section{Resultados}

La relación entre los cuerpos académicos y el porcentaje de profesores pertenecientes al (SNI), refleja situaciones complejas con respecto a la producción científica en la UAEMéx, ya que en teoría, los profesores integrados al SNI deben contar con al menos el grado de Doctor en alguna rama del conocimiento, ser profesores de tiempo completo de alguna institución pública de educación superior, haber dirigido tesis de posgrado, haber publicado algún artículo en revistas indexadas o libro reconocido, así como participar constantemente en congresos científicos. Es decir, ser profesores que aporten al conocimiento científico, tecnológico, social y cultural, mediante la investigación científica o tecnológica, la formación de recursos humanos especializados, la divulgación de la ciencia y la tecnología, la creación de grupos de investigación, el desarrollo de infraestructura científica y tecnológica, así como el fomento de labores para vincular la actividad de investigación con los sectores público, social y privado. Para el periodo comprendido entre 2002 a 2017, los investigadores de la UAEMéx en promedio el $4.06 \%$ están integrados al Sistema Nacional de Investigadores, condición que ha variado ligeramente durante los últimos 16 años, según datos de las Agendas estadísticas de dicho periodo. 
Como puede observarse en la tabla 1 , de los investigadores reconocidos por el SNI en el año 2002 que fueron 100, en comparación con los 512 del último año. Respectivamente representan para el primer año del periodo $2.3 \%$ y $6.5 \%$ del último año del periodo.

\begin{tabular}{|c|r|r|r|}
\hline Año & \multicolumn{1}{|c|}{ Total académicos } & \multicolumn{1}{c|}{ SNI } & $\%$ \\
\hline 2002 & 4286 & 100 & 0.0233 \\
\hline 2003 & 4493 & 119 & 0.0265 \\
\hline 2004 & 4736 & 131 & 0.0277 \\
\hline 2005 & 4994 & 150 & 0.0300 \\
\hline 2006 & 5047 & 189 & 0.0374 \\
\hline 2007 & 5054 & 228 & 0.0451 \\
\hline 2008 & 5266 & 267 & 0.0507 \\
\hline 2009 & 5457 & 270 & 0.0495 \\
\hline 2010 & 5708 & 269 & 0.0471 \\
\hline 2011 & 5979 & 289 & 0.0483 \\
\hline 2012 & 6239 & 305 & 0.0489 \\
\hline 2013 & 6644 & 353 & 0.0531 \\
\hline 2014 & 7181 & 418 & 0.0582 \\
\hline 2015 & 7467 & 463 & 0.0620 \\
\hline 2016 & 7595 & 484 & 0.0637 \\
\hline 2017 & 7797 & 512 & 0.0657 \\
\hline Promedio del periodo & & 0.0461 \\
\hline Coeficiente de correlación & & $98.44 \%$ \\
\hline
\end{tabular}

Tabla 1 Datos del personal académico UAEMéx Periodo: 2002-2017

Fuente: Elaboración propia y con base en las agendas estadísticas de la UAEMéx 2002 - 2013

El Sistema Nacional de Investigadores tiene un alto renombre que nos indica la calidad de educación con la que debe contar las personas adheridas a éste. Con esto nos referimos a que, el porcentaje de dichos profesores que pertenecen a la UAEMéx es una proporción que se sugiere debe incrementarse para favorecer la producción científica de la propia institución y por consiguiente su propio nivel académico.

En la tabla 2 se relacionan los datos de los cuerpos académicos, en la que se puede observar la evolución que han tenido los tres tipos de cuerpos: consolidado, en consolidación y en formación.

Se destaca como los cuerpos académicos en formación tienen la mayor proporción con relación a los otros cuerpos a lo largo del periodo. El crecimiento porcentual más alto durante el periodo de doce años, lo han tenido los cuerpos consolidados.

Considérese, que los datos recopilados se tomaron de las agendas estadísticas que han sido públicas por la propia Institución y que, además, se encuentran disponibles en el portal de la institución de referencia.

\begin{tabular}{|c|c|c|c|c|}
\hline Año & $\begin{array}{c}\text { Total } \\
\text { académicos }\end{array}$ & $\begin{array}{c}\text { Cuerpo } \\
\text { Académico } \\
\text { Consolidado }\end{array}$ & $\begin{array}{c}\text { Cuerpo } \\
\text { Académico en } \\
\text { Consolidación }\end{array}$ & $\begin{array}{c}\text { Cuerpo } \\
\text { Académico } \\
\text { en } \\
\text { Formación }\end{array}$ \\
\hline 2002 & 4286 & - & 9 & 56 \\
\hline 2003 & 4493 & - & 7 & 80 \\
\hline 2004 & 4736 & - & 7 & 85 \\
\hline 2005 & 4994 & 1 & 8 & 94 \\
\hline 2006 & 5047 & 5 & 20 & 71 \\
\hline 2007 & 5054 & 5 & 24 & 71 \\
\hline 2008 & 5266 & 5 & 31 & 57 \\
\hline 2009 & 5457 & 12 & 38 & 53 \\
\hline 2010 & 5708 & 19 & 40 & 62 \\
\hline 2011 & 5979 & 23 & 35 & 74 \\
\hline 2012 & 6239 & 30 & 47 & 63 \\
\hline 2013 & 6644 & 36 & 46 & 71 \\
\hline 2014 & 7181 & 36 & 46 & 74 \\
\hline 2015 & 7467 & 56 & 62 & 70 \\
\hline 2016 & 7595 & 63 & 72 & 73 \\
\hline 2017 & 7797 & 71 & 78 & 71 \\
\hline Promedio & 5871.44 & 27.85 & 35.63 & 70.31 \\
\hline del periodo & & & & \\
\hline Desviación estándar & 1156.68 & 23.57 & 22.65 & 10.68 \\
\hline del periodo & & & & \\
\hline
\end{tabular}

Tabla 2 Datos de Cuerpos académicos en la UAEMéx, Periodo: 2002-2017

Fuente: elaboración propia con base en las agendas estadísticas de la UAEMéx $2002-2013$

Como se observa, ha existido un crecimiento constante en los tres tipos de cuerpos académicos reconocidos por la secretaria de Educación Pública Federal (SEP), en el que para los cuerpos académicos consolidados el promedio del periodo es de 27 , para los cuerpos académicos en consolidación es de 35 y para los cuerpos académicos en formación es de 70. Se destaca el crecimiento que tienen los diferentes cuerpos a lo largo del periodo considerado, para lo cual se muestra la contrastación del promedio de todo el periodo con respecto al último año 2017.

\begin{tabular}{|c|c|c|}
\hline Año & Investigadores & $\begin{array}{l}\text { Proyectos de } \\
\text { Investigación }\end{array}$ \\
\hline 2002 & 210 & 285 \\
\hline 2003 & 215 & 346 \\
\hline 2004 & 255 & 476 \\
\hline 2005 & 265 & 614 \\
\hline 2006 & 286 & 655 \\
\hline 2007 & 300 & 657 \\
\hline 2008 & 323 & 855 \\
\hline 2009 & 629 & 871 \\
\hline 2010 & 729 & 993 \\
\hline 2011 & 833 & 823 \\
\hline 2012 & 805 & 873 \\
\hline 2013 & 753 & 802 \\
\hline 2014 & 794 & 874 \\
\hline 2015 & 815 & 596 \\
\hline 2016 & 915 & 635 \\
\hline 2017 & 978 & 649 \\
\hline \multicolumn{2}{|c|}{ Promedio del periodo } & 687.75 \\
\hline \multicolumn{2}{|c|}{ Coeficiente de correlación } & $55.17 \%$ \\
\hline
\end{tabular}

Tabla 3 Producción académica en la UAEMéx, Periodo: 2002-2013

Fuente: elaboración propia y con base en las agendas estadísticas de la UAEMéx 2002 - 2017 
En la tabla 3, se observa el crecimiento que ha tenido el número de investigadores integrantes en los cuerpos académicos descrito en la tabla 2 y el número de proyectos registrados por la propia institución. En dicha relación, anualmente el número de investigadores y número de proyectos de investigación presentan una tendencia creciente de crecimiento. El promedio de proyectos durante el periodo es de 688.

\section{Discusión}

La tabla 1. Muestra los datos del personal académico UAEMéx, periodo: 2002-2017, En ella se indica, la correlación que existe en los profesores adscritos y los pertenecientes al sistema nacional de investigadores. Esto es, en el año 2013 había 6644 profesores, de los cuales 353 son SNI. Sin embargo, solo el $6.57 \%$ cuenta con esta distinción. Aun cuando la correlación es alta $94.6 \%$, no es nada favorecedor para la universidad: Por lo cual habría que pensarse en instrumentar planes correctivos para mejorar esta condición.

En la tabla 2. Se presentan los datos de Cuerpos académicos en para el Periodo: 20022017., con respecto al estatus de integración y registro. Es decir, se analizaron los registros del total de académicos adscritos a la Universidad, los Cuerpo Académicos Consolidados, los Cuerpos Académico en Consolidación y los Cuerpos Académicos en Formación.

Reflejando que en 2013 se tenían 6644 profesores, pero solo había 36 cuerpos consolidados, 46 en consolidación y 71 en formación, lo cual representa un coeficiente de correlación de $99.1 \%$, para el primer grupo, 91.2\% para el segundo y un $-0.18 .7 \%$ para la tercera categoría. Por tanto, habría que observar detenidamente estas cifras ya que indican que la mayoría de los docentes está ubicada en cuerpos en formación.

La tabla 3. Presenta la producción académica en la UAEMéx, periodo: 2002-2017. En ella se analizan los datos de los investigadores reconocidos por la UAEMéx y el total de investigaciones registradas.

En el último periodo de 2017 las cifras muestran 649 investigaciones.

\section{Conclusiones}

El crecimiento en el número de los investigadores registrados en el SNI ha incidido en el crecimiento de los diversos tipos de cuerpos académicos de la universidad registrados en la SEP. Esta tendencia se refleja como un referente institucional, pues presenta una serie de situaciones intrínsecas que expresan las posibilidades de generación de conocimiento. El incremento de los cuerpos académicos presenta una tendencia creciente durante el periodo analizado. Sin embargo, sería interesante analizar en un estudio de naturaleza cualitativo la relación entre la producción científica y las necesidades académicas de la institución. La relación entre número de investigadores y proyectos de investigación generados por los cuerpos académicos tiene una tendencia creciente año con año, lo cual garantiza la continuidad de profesores en el SNI, así como la producción y generación de conocimiento.

\section{Referencias}

CONACYT. (2014). Sistema Nacional de Investigadores. Consultado el 09/04/2014 en: http://www.conacyt.mx/index.php/elconacyt/sistema-nacional-de-investigadores

Hernández, R. Fernández, C y Baptista, P. (2010). Metodología de la Investigación. México. Mc Graw Hill.

Siegel S. y Castllan, J. (2009). Estadística no paramétrica aplicada a las Ciencias de la conducta. México. Editorial Trillas.

Tejedor, F. y Etxeberria, J. (2006). Análisis inferencial de datos en educación. Madrid, España. Editorial la Muralla.

UAEMéx. Agendas estadísticas 2002 a 2017. Consultado el 24/04/2017 en: http://www.uaemex.mx/planeacion/ 